\title{
Do Elevated Triglycerides Truly Trigger Acute Pancreatitis?
}

\author{
Mahya Faghih ${ }^{1}$. Vikesh K. Singh ${ }^{1,2}$
}

Published online: 6 February 2019

(c) Springer Science+Business Media, LLC, part of Springer Nature 2019

Hypertriglyceridemia, the third most common cause of acute pancreatitis (AP) in the USA, has recently been reported to be the most common cause of AP in China [1]. There is an increased risk of severe acute pancreatitis in patients with hypertriglyceridemic AP, with a recent prospective study using trend analysis across patients with normal $(<150 \mathrm{mg} /$ $\mathrm{dL}$ ) to severe ( $\geq 1000 \mathrm{mg} / \mathrm{dL})$ TG levels reporting significantly higher rates of persistent organ failure, need for ICU care, and longer length of hospital stay with rising triglyceride levels [2]. While a TG level of $\geq 1000 \mathrm{mg} / \mathrm{dL}$ is considered the threshold at which the risk of AP increases, the absolute risk is still low at $5 \%$, which modestly increases to $10-20 \%$ in patients with TG levels $>2000 \mathrm{mg} / \mathrm{dL}$ [3]. Nonetheless, the risk of AP is not negligible at lower TG levels; a large epidemiologic study from Denmark reported increased risk of AP starting when TG levels were $>177 \mathrm{mg} /$ $\mathrm{dL}$ (HR 2.3; 95\% CI 1.3-4.0) that linearly increased to a HR 7.7; 95\% CI 3-19.8 when TG levels were $>443$ mg/dL $[4,5]$.

Elevated TG levels ( $\geq 150 \mathrm{mg} / \mathrm{dL}$ ) are present in nearly one-quarter, and highly elevated TG levels (500-2000 mg/ dL) are present in $1.7 \%$ of the US adult population [6]. The development and degree of hypertriglyceridemia are governed by both primary (genetic) and secondary factors (e.g., uncontrolled diabetes, alcohol use, high-fat diet, obesity, pregnancy, medications) that are common in the general population [7]. Yet, it is not known why relatively few among the many individuals with hypertriglyceridemia develop AP. Among susceptible patients such as those with baseline elevated TG levels who develop AP, the risk is multifactorial and likely based on the presence and interplay of primary and secondary factors, similar to the interplay

Vikesh K. Singh

vsingh1@jhmi.edu

1 Division of Gastroenterology, Pancreatitis Center, Johns Hopkins Medical Institutions, 1830 E. Monument Street, Room 428, Baltimore, MD 21205, USA

2 Department of Medicine, Johns Hopkins Medical Institutions, Baltimore, MD, USA of primary and secondary factors thought contributory to the etiology of other forms of AP. For example, the lifetime risk of AP in patients with heavy alcohol use is 4\% [8], with smoking it is $1.8 \%$ [9], and with pancreas sufficient cystic fibrosis it is $22.4 \%$ [10]. The risk of alcoholic pancreatitis is higher in patients with pancreatitis-associated genetic variants such as serine protease inhibitor Kazal-type (SPINK)1 $\mathrm{N} 34 \mathrm{~S}$ [11] as well as risk-modifying genetic variants such as claudin (CLDN)2 [12]. It is certainly possible that patients with hypertriglyceridemic AP carry similar genetic variants.

In this issue of Digestive Diseases and Sciences, Wu and colleagues [13] present the results of a retrospective study evaluating the follow-up TG levels and the associated risk of AP in outpatients who were previously hospitalized for hypertriglyceridemic AP. The study cohort consisted of 151 patients who had TG levels $\geq 500 \mathrm{mg} / \mathrm{dL}$ during hospitalization for AP in the Kaiser Permanente Southern California region. All patients had at least one outpatient TG level measured within 1 year following their index hospitalization for AP and were followed from the date of their outpatient TG level measurement to a recurrence of AP. The distributions of outpatient TG levels were low normal (0-200 mg/ $\mathrm{dL})$ in $31 \%$, moderately elevated (200-500 $\mathrm{mg} / \mathrm{dL})$ in $44 \%$, and highly elevated ( $>500 \mathrm{mg} / \mathrm{dL}$ ) in $24.5 \%$. Approximately $2 / 3$ of the patients had a TG level measured within 30 days of hospital discharge. Over a median follow-up of 3 years, $45(29.8 \%)$ patients experienced at least one recurrent episode of AP, and among these patients, 25 (55\%) experienced multiple recurrent episodes of AP. Patients with higher outpatient TG levels had a greater subsequent risk of recurrent AP. After adjusting for age, gender, diabetes, and alcohol use, patients who continued to have moderately elevated (RR 5.47; 95\% CI 1.80-16.65) as well as highly elevated (RR 8.45; 95\% CI 2.55-27.96) TG levels were at an increased risk of recurrent AP as compared to patients that achieved normalization of their TG levels.

This study further supports the correlation between TG levels and the risk of AP and suggests that reducing TG levels can reduce the risk of AP in susceptible individuals. There are several obvious limitations mentioned by the 
authors including small sample size, short follow-up period, and selection bias, since nearly $57 \%$ of their inpatients with AP had no TG level measured during hospitalization. Nevertheless, there were additional limitations. The first is the use of nonstandard definitions for the categorization of TG levels. The use of a TG level of $>500 \mathrm{mg} / \mathrm{dL}$ as the cutoff for "highly elevated" prevents further stratification of the study cohort into more commonly accepted thresholds for AP risk, i.e., TG $>1000$ and $>2000 \mathrm{mg} / \mathrm{dL}$. The second is the lack of additional details regarding the management of secondary factors that could result in hypertriglyceridemic AP. Since nearly $28 \%$ of the study cohort had alcohol as the etiology of AP that resulted in hypertriglyceridemia, it would follow that prevention of further alcohol use could potentially prevent recurrence of AP. Similarly, glycemic control for uncontrolled diabetes, weight loss for obesity, and dietary modification in those individuals ingesting high-fat diets could also reduce the risk of AP without the need for anti-lipidemic therapy. This point carries particular importance if TG lowering strategies remain focused on secondary prophylaxis. The third is that it is not clear if the outpatient TG levels were measured in the fasting or in the nonfasting state. Fasting TG assessments are advocated by professional society guidelines, but it is nonfasting levels that drive the risk of AP [14].

There are larger issues that continue to challenge clinicians evaluating patients with hypertriglyceridemia across the general population. The first is that TG levels remain a poor screening marker for the risk of AP. As stated above, the absolute risk of AP is low in even patients with TG levels $>1000 \mathrm{mg} / \mathrm{dL}$. The second is that the risk of AP can vary over time regardless of TG level. This suggests that studies are needed to better understand the risk of AP based on longitudinal triglyceride measurements as well as an assessment of primary and secondary factors across the general population. Since the risk and timing of AP in hypertriglyceridemia are not known, prior studies that have evaluated hypertriglyceridemic patients over short periods of time (i.e., months to a year) may detect few to no AP events. This is the likely explanation for a lack of a significant difference in the number of AP events between the treatment and placebo arms of a trial evaluating a novel antisense apoC-III inhibitor across patients with both primary and secondary hypertriglyceridemia [15]. Once genetic testing for disorders of lipid metabolism and for pancreatitis is more widely available and less expensive, their use will potentially help define which hypertriglyceridemic patients require primary prophylaxis against AP with lipid-lowering therapies and secondary risk factor modification. These primary prophylaxis strategies may be implemented at lower TG levels than that are currently being advocated for the prevention of cardiovascular complications associated with hypertriglyceridemia. There are some examples of this already taking place in clinical practice without genetic testing. Baseline TG level assessment can be helpful in patients who are treated with medications that can significantly increase TG levels (e.g., steroids or antiretroviral therapy) as well as women with visceral obesity or those with family history of dyslipidemia who are contemplating the use of exogenous estrogen as both groups of patients are at an increased risk of AP [14].

In summary, the incidence of hypertriglyceridemic AP has been increasing over the past few years. While elevated TG levels are associated with an increased risk of AP, most individuals with elevated TG will not develop AP, and the TG level at which AP is triggered can vary in susceptible patients who have experienced prior episodes of AP. In addition to reducing triglyceride levels through lifestyle changes and lipid-lowering medications, future precision medicinebased screening approaches will be required to decrease the risk of pancreatitis.

\section{Compliance with ethical standards}

Conflict of interest Vikesh K. Singh is a consultant to Abbvie, Ariel Precision Medicine, Orgenesis and Akcea Therapeutics. These financial interests do not pertain directly to this work. Mahya Faghih has no disclosures.

\section{References}

1. Zhu Y, Pan X, Zeng H, et al. A study on the etiology, severity, and mortality of 3260 patients with acute pancreatitis according to the Revised Atlanta Classification in Jiangxi, China over an 8-year period. Pancreas. 2017;46:504-509.

2. Nawaz H, Koutroumpakis E, Easler J, et al. Elevated serum triglycerides are independently associated with persistent organ failure in acute pancreatitis. Am J Gastroenterol. 2015;110:1497-1503.

3. Scherer J, Singh VP, Pitchumoni CS, Yadav D. Issues in hypertriglyceridemic pancreatitis: an update. J Clin Gastroenterol. 2014;48:195-203.

4. Hansen SEJ, Madsen CM, Varbo A, Nordestgaard BRG. Lowgrade inflammation in the association between mild-to-moderate hypertriglyceridemia and risk of acute pancreatitis: a study of more than 115,000 individuals from the general population. Clin Chem. 2018;65:321-332.

5. Pedersen SB, Langsted A, Nordestgaard BG. Nonfasting mildto-moderate hypertriglyceridemia and risk of acute pancreatitis. JAMA Int Med. 2016;176:1834-1842.

6. Christian JB, Bourgeois N, Snipes R, Lowe KA. Prevalence of severe (500 to $2000 \mathrm{mg} / \mathrm{dl}$ ) hypertriglyceridemia in United States adults. Am J Cardiol. 2011;107:891-897.

7. Hegele RA, Ginsberg HN, Chapman MJ, et al. The polygenic nature of hypertriglyceridaemia: implications for definition, diagnosis, and management. Lancet Diabetes Endocrinol. 2014;2:655-666.

8. Yadav D, Hawes RH, Brand RE, et al. Alcohol consumption, cigarette smoking, and the risk of recurrent acute and chronic pancreatitis. Arch Intern Med. 2009;169:1035-1045. 
9. Majumder S, Gierisch JM, Bastian LA. The association of smoking and acute pancreatitis: a systematic review and meta-analysis. Pancreas. 2015;44:540-546.

10. Ooi CY, Dorfman R, Cipolli M, et al. Type of CFTR mutation determines risk of pancreatitis in patients with cystic fibrosis. Gastroenterology. 2011;140:153-161.

11. Di Leo M, Bianco M, Zuppardo RA, et al. Meta-analysis of the impact of SPINK1 p.N34S gene variation in Caucasic patients with chronic pancreatitis. An update. Dig Liver Dis. 2017;49:847-853.

12. Derikx MH, Kovacs P, Scholz M, et al. Polymorphisms at PRSS1PRSS2 and CLDN2-MORC4 loci associate with alcoholic and non-alcoholic chronic pancreatitis in a European replication study. Gut. 2015;64:1426-1433.

13. Wu BU, Batech M, Dong EY, Duan L, Yadav D, Chen W. Influence of ambulatory triglyceride levels on risk of recurrence in patients with hypertriglyceridemic pancreatitis. Dig Dis Sci. (Epub ahead of print). https://doi.org/10.1007/s10620-018-5226-x.

14. Driver SL, Martin SS, Gluckman TJ, Clary JM, Blumenthal RS, Stone NJ. Fasting or nonfasting lipid measurements: it depends on the question. J Am Coll Cardiol. 2016;67:1227-1234.

15. Gaudet D, Alexander VJ, Baker BF, et al. Antisense inhibition of apolipoprotein C-III in patients with hypertriglyceridemia. $N$ Engl J Med. 2015;373:438-447.

Publisher's Note Springer Nature remains neutral with regard to jurisdictional claims in published maps and institutional affiliations. 